\title{
The Effect of Ciprofloxacin on the Pharmacokinetic and ECG Parameters of Quinidine
}

\author{
Barry E. Bleske, PharmD, Peggy L. Carver, PharmD, Thomas M. Annesley, PhD, \\ Jane R. M. Bleske, RN, and Fred Morady, MD
}

\begin{abstract}
Ciprofloxacin decreases the clearance of antipyrine and other drugs which, in part, undergo oxidative metabolism. Based on these findings, the authors hypothesized that ciprofloxacin may decrease the clearance of quinidine, a drug which also undergoes oxidative metabolism. The purpose of this study was to evaluate the effect of ciprofloxacin on the pharmacokinetic and ECG parameters of quinidine in seven healthy men. Oral quinidine sulfate $400 \mathrm{mg}$ was administered alone (Phase A) and after oral ciprofloxacin pretreatment (Phase B) in a randomized crossover fashion with a 2-week washout period between each phase. During Phase B, ciprofloxacin pretreatment $(750 \mathrm{mg}$ every 12 hours) was administered for 5 days before and 24 hours after quinidine administration. Quinidine serum samples were obtained over a 24-hour period. QRS and QTC intervals were measured over a 12-hour period. There were no significant differences in clearance $(20.3 \pm 3.3 \mathrm{~L} / \mathrm{hr}$ vs $20.1 \pm 2.3 \mathrm{~L} / \mathrm{hr}, \mathrm{P}=.836)$, half-life $(7.9 \pm 1 \mathrm{hr}$ vs $7.8 \pm 0.8 \mathrm{hr}$, $P=0.8)$, maximum concentration $(1.4 \pm 0.6 \mathrm{mg} / \mathrm{L}$ vs $1.5 \pm 0.6 \mathrm{mg} / \mathrm{L}, \mathrm{P}=0.613)$, or time to maximum concentration ( $1.5 \pm 0.2 \mathrm{hr}$ vs $1.5 \pm 0.1 \mathrm{hr}, \mathrm{P}=0.571)$ for quinidine between Phase $A$ and Phase $B$, respectively. The largest decrease in clearance observed for Phase $B$ compared to Phase $A$ was $10 \%$. There was also no significant difference in the degree of QRS and QTc prolongation between Phase $A$ and Phase B. From these results, it appears that ciprofloxacin in the dose given does not alter the pharmacokinetic or ECG parameters of quinidine. Therefore, no adjustment in the dose of quinidine is needed when coadministered with ciprofloxacin.
\end{abstract}

\begin{abstract}
Ciprofloxacin is an orally available fluoroquinolone antibiotic with a broad spectrum of grampositive and gram-negative activity. It is used for the treatment of a wide variety of infections, including pneumonia, skin and soft tissue, urinary tract, and other serious infections. Ciprofloxacin is eliminated in part by oxidative pathways; the M3 metabolite of ciprofloxacin comprises approximately $9.6 \%$ of the urinary excretion of ciprofloxacin following an oral dose of $250 \mathrm{mg}$ in healthy volunteers. ${ }^{1}$ Therefore, it

From the University of Michigan, College of Pharmacy (Barry Bleske) and University of Michigan Medical Center Departments of Pharmacy (Peggy Carver), Pathology (Thomas Annesley), and Internal Medicine (Fred Morady). This study was supported in part by the Research Fund, College of Pharmacy, University of Michigan and CRC grant 5MO1RR00042 through the National Institutes of Health. Address for reprints: Barry E. Bleske, PharmD, Assistant Professor of Pharmacy, University of Michigan, College of Pharmacy, Ann Arbor, MI 481091065.
\end{abstract}

is not surprising that ciprofloxacin has been shown to decrease the metabolic clearance of caffeine, theophylline, and antipyrine, which also undergo hepatic oxidation..$^{2-6}$

Because ciprofloxacin is widely used in both the community and hospital settings, there is a potential for concurrent administration with other drugs that are eliminated by oxidative pathways such as caffeine or theophylline. Quinidine also undergoes oxidative metabolism, and its clearance is affected by inhibitors of oxidative metabolism such as cimetidine, propranolol, and verapamil. ${ }^{7-11}$ Like ciprofloxacin, these agents also decrease the clearance of antipyrine. ${ }^{12-14}$ Therefore, it is likely that ciprofloxacin will also decrease the clearance of quinidine. Since ciprofloxacin and quinidine are widely used, concomitant administration of both agents is likely to occur.

Quinidine is an antiarrhythmic agent with a narrow therapeutic range; serum concentrations are 
usually maintained between 2 and $5 \mathrm{mg} / \mathrm{L}$ for the control of atrial and ventricular arrhythmias. The narrow therapeutic range of quinidine suggests that any reduction in the clearance of quinidine is likely to be of pharmacokinetic and pharmacodynamic significance. Therefore, the purpose of this study was to determine the effect of ciprofloxacin coadministration on the pharmacokinetic and ECG parameters of quinidine.

\section{METHODS}

Eight healthy nonsmoking male subjects between the ages of 22 and 34 years were enrolled in the study. Each subject was considered healthy based upon history, physical examination, ECG, blood chemistry, and hematological screen. All subjects were within $20 \%$ of ideal body weight. Subjects were asked to refrain from taking any drugs (including over-the-counter products), or xanthine-containing foods (including caffeine) $\mathbf{4 8}$ hours before each phase of the study. Subjects who had taken any drugs known to induce liver enzymes within the past 30 days were excluded from the study. The study was approved by The University of Michigan Institutional Review Board and written informed consent was obtained from all subjects. The study was performed in the Clinical Research Center.

The study consisted of two phases with a 2-week washout period between phases. In a randomized crossover design, subjects received a $400 \mathrm{mg}$ oral dose of quinidine sulfate (Warner Chilcott, Morris Plains, NJ) alone or after pretreatment with oral ciprofloxacin (Miles Inc., West Haven, CT) $750 \mathrm{mg}$ at 7:00 AM and 7:00 PM for 5 days. Quinidine was administered with $240 \mathrm{~mL}$ of water after an overnight fast and blood samples were obtained from an indwelling venous catheter in an antecubital vein at 0 (predose), 0.5, 1.0, 1.5, 2.0, 2.5, 3.0, 4.0, 6.0, 8.0, 10.0, 12.0, and 24.0 hours after administration of quinidine. During the treatment phase of the study, ciprofloxacin was administered 30 minutes before the quinidine dose after an overnight fast. Ciprofloxacin was administered throughout the 24-hour quinidine sampling period as previously scheduled. During the first 12 hours of serum sampling, single lead electrocardiogram monitoring was performed with a cardiac monitor (Mennem Medical 741, Clarence NY) after each blood sample was obtained. Subjects were provided a standard meal after the 4.0 and 8.0 hour blood samples. Blood samples were drawn into unheparinized vacuum tubes (Becton Dickinson, Rutherford NJ) and allowed to clot. After centrifugation the serum was harvested and stored at $-20^{\circ} \mathrm{C}$ pending analysis.

\section{Drug Analysis}

Quinidine serum concentrations were determined by a fluorescence polarization method (TDx, Abbott Laboratories, North Chicago, IL). This method has been reported to be specific for quinidine. ${ }^{11,15}$ The coefficient of variation was less than $5 \%$ over the concentration range of 0.05 to $2.0 \mathrm{mg} / \mathrm{L}$. The serum free fraction of quinidine was determined by ultrafiltration (Amicon Centrifree Micropartition System, Amicon Corp., Danvers, MA) of the serum samples obtained at 2.0 hours after administration of quinidine. The $\mathrm{pH}$ of the ultrafiltrate was adjusted to a physiological $\mathrm{pH}$ of approximately 7.40 with $1 \mathrm{M}$ phosphoric acid prior to analysis. ${ }^{16.17}$ Ciprofloxacin serum concentrations were determined by a microbiological assay employing Bacillus subtilis ATCC 6633 as the test organism.

\section{Data Analysis}

The apparent oral clearance of quinidine was calculated by dividing the administered dose in terms of quinidine base by the area under the serum concentration-time curve from zero to infinity (AUC). The AUC was calculated using the linear trapezoidal method. The elimination half-life $\left(t_{1 / 2}\right)$ was determined by linear regression analysis of the terminal phase of the log concentration-time profile. The mean maximal serum concentration $\left(\mathrm{C}_{\max }\right)$ and time to $C_{\max }\left(t_{\max }\right)$ was determined by visual inspection of the available data points.

The QRS and QTc intervals were measured by a trained nurse who was blinded to the study. The QRS and QTc intervals were determined over a 10beat interval and are reported as average values. The QTc interval was calculated by dividing the QT interval by the square root of the R-R interval. The variability in measuring the ECG intervals was evaluated by determining the mean percentage of difference from repeated measurements of a random sampling of ECG parameters. The intraperson percent difference was less than $6 \%$ for all ECG parameters measured. A random sample of ECG parameters were also measured by a second trained person who was also blinded; the interperson mean percent difference for the ECG parameters measured was less than $8 \%$. The area under the electrocardiographic parameter percent change versus time curve (AUCE) was determined for each subject from 0-12 hours using the linear trapezoidal method. ${ }^{9}$

Statistical comparison between the two phases was performed using a two-tailed paired Student's $t$ test. A $P$ value of less than .05 was considered statis- 


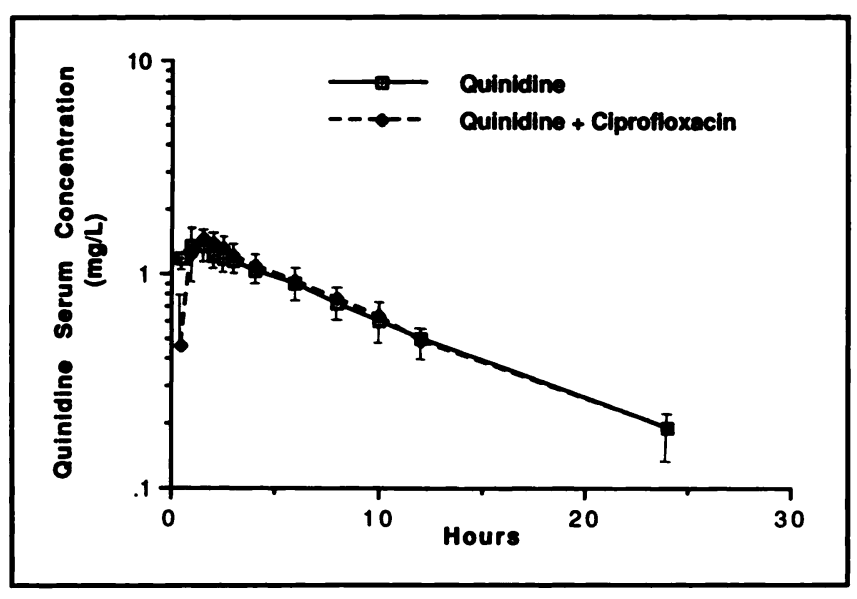

Figure 1. Quinidine serum concentration versus time curve for quinidine and quinidine plus ciprofloxacin phases. (Mean and standard deviation).

tically significant. The reported data are represented as the mean and standard deviation unless otherwise specified.

\section{RESULTS}

Seven of the eight patients completed both phases of the study. One patient withdrew from the study secondary to development of a rash after ciprofloxacin administration. All subjects were compliant with the ciprofloxacin regimen as assessed by patient questioning.

The mean serum quinidine concentration versus time profile for each phase is presented in Figure 1. The pharmacokinetic data for each subject are summarized in the Table. Quinidine clearance was not significantly altered after ciprofloxacin administration, $20.3 \pm 3.3 \mathrm{~L} / \mathrm{hr}$ vs $20.1 \pm 2.3 \mathrm{~L} / \mathrm{hr}(P=.836)$ for the control (quinidine alone) and treatment phases (quinidine and ciprofloxacin) respectively. The maximal percent decrease in clearance from the control phase to treatment phase was $10 \%$, with a mean decrease of $8.5 \pm 2.0 \%$ in four out of the seven patients demonstrating a decrease in quinidine clearance. Overall, there was less than a $1 \%$ decrease in quinidine clearance between the control and treatment phases. There was no statistical difference in the half-life between the two phases $(P$ $=.8$ ).

There was no statistical difference in $\mathrm{C}_{\max }, \mathrm{t}_{\max }$, or free fraction of quinidine between control and treatment phases. The $\mathrm{C}_{\max }$ values during control and treatment phases were $1.4 \pm 0.6 \mathrm{mg} / \mathrm{L}$ and $1.5 \pm 0.6$ $\mathrm{mg} / \mathrm{L}(P=.613)$, respectively and $t_{\max }$ values were
$1.5 \pm 0.2$ hours vs $1.5 \pm 0.1$ hours $(P=.571)$ for the control and treatment phases, respectively. The free fraction of quinidine was similar between the control and treatment phase, $21 \pm 5.5 \%$ vs. $20 \pm 3.5 \%$, respectively.

The percentage of change from baseline in the QRS and QTc interval during each time period measured is shown in Figures 2 and 3. The AUC-E for the QRS and QTc interval was not significantly different between the control and treatment phases. The maximal percent increase in the QRS interval from baseline occurred at 2.0 hours for the control and treatment phase, $12.1 \pm 4 \%$ vs. $10.7 \pm 6.7 \%$, respectively (mean and standard error). The maximal percent increase in the QTc interval from baseline occurred at 1.5 hours for the control and treatment phase, $17 \pm 3.1 \%$ vs. $15 \pm 2.8 \%$, respectively (mean and standard error).

The concentration of ciprofloxacin in serum 2 hours after dosing with quinidine averaged 2.83 $\pm 0.12 \mathrm{mg} / \mathrm{L}$.

\section{DISCUSSION}

The current study evaluated the influence of ciprofloxacin on the pharmacokinetic and ECG parameters of quinidine. The pharmacokinetic parameters of quinidine during the control phase and the ciprofloxacin serum concentration observed during the treatment phase of this study was consistent with previous reports in the literature..$^{9,11,18,19}$ In this study the oral clearance, $t_{1 / 2}, C_{\max }$, and $t_{\max }$ were not altered by ciprofloxacin administration. The mean decrease in quinidine clearance was less than $1 \%$ and no subject experienced a greater than $10 \%$ decrease in quinidine clearance during the treatment

\section{TABLE}

Quinidine Pharmacokinetic Parameters Before and After Ciprofloxacin Administration

\begin{tabular}{cccccc}
\hline & \multicolumn{2}{c}{ Oral Clearance (L/hr) } & & \multicolumn{2}{c}{$t_{1 / 2}$ (hr) } \\
\cline { 2 - 3 } \cline { 5 - 6 } Subject & Quinidine & $\begin{array}{c}\text { Quinidine } \\
\text { + Ciprofiloxacin }\end{array}$ & & Quinidine & $\begin{array}{c}\text { Quinidine } \\
\text { + Ciprofioxacin }\end{array}$ \\
\hline 1 & 19.9 & 18.0 & & 8.2 & 8.2 \\
2 & 20.1 & 20.8 & & 7.8 & 8.3 \\
3 & 20.4 & 19.2 & & 9 & 8.2 \\
4 & 23.8 & 21.8 & & 7.1 & 6.5 \\
5 & 23.8 & 21.4 & & 8 & 8.3 \\
6 & 20.2 & 23.0 & & 6.1 & 6.7 \\
7 & 13.6 & 16.3 & & 9 & 8.4 \\
Mean \pm SD & $20.3 \pm 3.3$ & $20.1 \pm 2.3$ & $7.9 \pm 1$ & $7.8 \pm 0.8$ \\
\hline
\end{tabular}




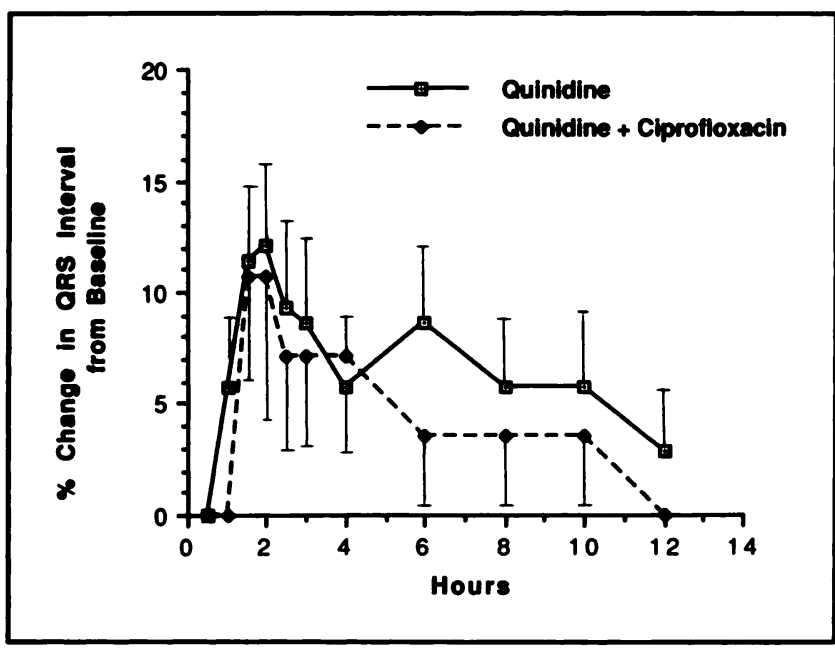

Figure 2. Percentage of change in the QRS interval from baseline over a 12-hour time period for quinidine and quinidine plus ciprofloxacin phases. (Mean and standard error).

phase compared to the control phase. In both phases the QRS and QTc interval increased during the study period, consistent with quinidine's electrophysiological properties. In agreement with the pharmacokinetic findings, there was no obvious difference in the degree of QRS or QTc prolongation between the treatment and control phases.

Based on recent studies with theophylline, caffeine, and antipyrine suggesting that ciprofloxacin may interfere with oxidative metabolism, it was expected that ciprofloxacin would decrease the clearance of quinidine.$^{2-6}$ Surprisingly, the results of the study demonstrated that ciprofloxacin had no effect on the clearance of quinidine. From these results it is clear that the effect of ciprofloxacin on antipyrine or theophylline clearance cannot always be extrapolated to other drugs. ${ }^{20} \mathrm{Ciprofloxacin}$ may inhibit specific oxidative pathways responsible for antipyrine, theophylline, or caffeine metabolism. Therefore, substrate specificity for different forms of cytochrome P-450 by ciprofloxacin may account for the drug interactions that are observed with antipyrine, theophylline, and caffeine but not for quinidine.

Although, the specific isoenzymes inhibited by ciprofloxacin are not known, some generalizations can be made. Quinidine is metabolized apparently in part by the cytochrome $\mathrm{P}-450_{\mathrm{NF}}$ isoenzyme. ${ }^{21}$ Since quinidine clearance was not affected by ciprofloxacin administration it can be postulated that ciprofloxacin does not interfere with the metabolism of drugs which undergo metabolism by the cytochrome $\mathrm{P}-450_{\mathrm{NF}}$ isoenzyme and which have a sim- ilar affinity for the isoenzyme as quinidine. In support of this a recent study showed that the synthesis of testosterone, which is affected by the P-450 isoenzyme, was not inhibited by ciprofloxacin administration. ${ }^{22}$ In contrast, ciprofloxacin does appear to inhibit the isoenzymes responsible for the metabolism of theophylline, caffeine, and antipyrine. However, it is unknown which specific isoenzymes and metabolic pathways of these drugs are inhibited by ciprofloxacin. Further studies with in vitro models involving specific human isoenzymes are necessary to determine the relationship between ciprofloxacin and drug metabolism.

Other possible reasons for a negative study include dose and duration of drug therapy or Type II error. Ciprofloxacin was administered over a 6-day period at a dose of $750 \mathrm{mg}$ twice a day. The time period over which ciprofloxacin was administered would allow for achievement of steady state levels of ciprofloxacin and is in agreement with the time periods used in other studies evaluating interactions with antipyrine and theophylline. ${ }^{2-5}$ The dose evaluated is the maximum recommended dose and if a drug interaction is to occur it should be apparent at this dose. Therefore, it is unlikely that the dose or duration of ciprofloxacin therapy used in this study would result in a negative study. A clinically significant Type II error is also unlikely to account for a negative study. This study should be able to detect an $18 \%$ change in quinidine clearance based on a power value of 0.8 and seven subjects. Any change in clearance less than $18 \%$ and particularly under

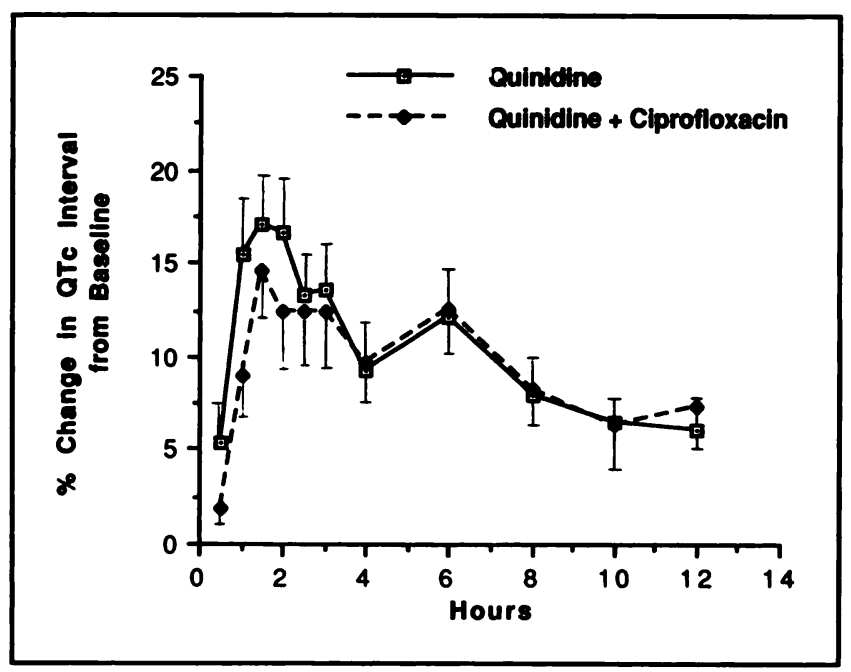

Figure 3. Percentage of change in the QTc interval from baseline over a 12-hour time period for quinidine and quinidine plus ciprofloxacin phases. (Mean and standard error). 
$10 \%$ is unlikely to be clinically relevant. Assuming linear pharmacokinetics for quinidine, a $10 \%$ change in the clearance of quinidine would result in an increase in average quinidine steady state serum concentrations of less than $11 \%$, which would not necessitate a dosage adjustment. No subject in this study demonstrated a change in quinidine clearance greater than $10 \%$ in the treatment phase as compared to the control phase.

What is not known from this study is the effect of other quinolone antibiotics on quinidine metabolism and clearance. Other quinolone antibiotics, particularly enoxacin which is extensively metabolized to a 4-oxo-quinolone metabolite may interfere with quinidine metabolism. The 4-oxo-quinolone metabolite has been postulated to be responsible for the inhibition of theophylline metabolism. ${ }^{2}$ However, the role of the 4-oxo-quinolone metabolite in interfering with drug metabolism is controversial. ${ }^{4,6.23}$ Additional studies are required to address this issue.

In conclusion, the results of this study showed that ciprofloxacin does not alter the pharmacokinetic or electrocardiographic parameters of quinidine. No dosage adjustments are required for quinidine when administered with ciprofloxacin. Previous studies demonstrating drug interactions with antipyrine and theophylline were not predictive with quinidine. Additional studies are required to determine if other quinolone antibiotics may cause significant drug interaction with quinidine.

The authors are grateful to Debbie Loughry for her secretarial assistance.

\section{REFERENCES}

1. Gau W, Kurz J, Petersen U, Ploschke HJ, Wuensche C: Isolation and structural elucidation of urinary metabolites of ciprofloxacin. Arzneim-Forsch/Drug Res 1986;36(II):1545-1549.

2. Wijnands WJA, Vree TB, Van Herwaarden CLA: The influence of quinolone derivatives on theophylline clearance. $\mathrm{Br} J$ Clin Pharmacol 1986;22:677-683.

3. Nix DE, DeVito JM, Whitbread MA, Schentag JJ: Effect of multiple dose oral ciprofloxacin on the pharmacokinetics of theophylline and indocyanine green. J Antimicrob Chemother 1987;19:263-269.

4. Prince RA, Casabar E, Adair CG, Wexler DB, Lettieri J, Kasik JE: Effect of quinolone antimicrobials on theophylline pharmacokinetics. J Clin Pharmacol 1989;29:650-654.

5. Ludwig E, Szekely E, Csiba A, Graber H: The effect of ciprofloxacin on antipyrine metabolism. J Antimicrob Chemother 1988;22:61-67.
6. Healy DP, Polk RE, Kanawati L, Rock DT, Mooney ML: Interaction between oral ciprofloxacin and caffeine in normal volunteers. Antimicrob Agents Chemother 1989;33:474-478.

7. Drayer DE, Lowenthal DT, Restivo KM, Schwartz A, Cook CE, Reidenberg MM: Steady-state serum levels of quinidine and active metabolites in cardiac patients with varying degrees of renal function. Clin Pharmacol Ther 1978;24:31-39.

8. Ueda CT: Quinidine, In Evans WE, Schentag JJ, Jusko WJ (eds): Applied Pharmacokinetics: Principles of Therapeutic Drug Monitoring. Spokane, Applied Therapeutics, Inc., 1986;000-000.

9. Hardy BG, Zador IT, Golden L, Lalka D, Schentag JJ: Effect of cimetidine on the pharmacokinetics and pharmacodynamics of quinidine. Am J Cardiol 1983;52:172-175.

10. Kessler KM, Humphries WC Jr., Black M, Spann JF: Quinidine pharmacokinetics in patients with cirrhosis or receiving propranolol. Am Heart J 1978;96:627-635.

11. Edwards DJ, Lavoie R, Beckman $H$, Blevins $R$, Rubenfire $M$ : The effect of coadministration of verapamil on the pharmacokinetics and metabolism of quinidine. Clin Pharmacol Ther 1987;41:68-73.

12. Greenblatt DJ, Franke $K$, Huffman DH: Impairment of antipyrine clearance in humans by propranolol. Circulation 1978;57:1161-1164.

13. Bax NDS, Lennard MS, Tucker GT: Inhibition of antipyrine metabolism by beta-adrenoceptor antagonists. $\mathrm{Br} J$ Clin Pharmacol 1981;12:779-784.

14. Bach DR, Blevins R, Kerner N, Rubenfire M, Edwards DJ: The effect of verapamil on antipyrine pharmacokinetics and metabolism in man. Br J Clin Pharmacol 1986;21:655-659.

15. Wolf-Rogers JA, Smith CM, Vaughn K: Automated fluorescence polarization immunoassay for monitoring quinidine (abstr). Clin Chem 1984;30:1020.

16. Chen BH, Taylor EH, Ackerman BH, Olsen K, Pappas AA: Effect of $\mathrm{pH}$ on free quinidine (letter). Drug Intell Clin Pharm 1988;22:826.

17. Verme CN, Ludden TM, Harris SC: Effect of temperature on in vitro measurement of quinidine free fraction. Clin Pharm 1988;7:142-146.

18. Tartaglione TA, Raffalovich AC, Poynor WJ, Espinel-Ingroff A, Kerkering TM: Pharmacokinetics and tolerance of ciprofloxacin after sequential increasing oral doses. Antimicrob Agents Chemother 1986;29:62-66.

19. Hoffken G, Lode H, Prinzing C, Borner K, Koeppe P: Pharmacokinetics of ciprofloxacin after oral and parenteral administration. Antimicrob Agents Chemother 1985;27:375-379.

20. Vesell ES: Commentary. The antipyrine test in clinical pharmacology: Conceptions and misconceptions. Clin Pharmacol Ther 1979;26:275-286.

21. Guengerich FP, Muller-Enoch D, Blair IA: Oxidation of quinidine by human liver cytochrome P-450. Mol Pharmacol 1986;30:287-295.

22. Waite NM, Edwards DJ, Arnott WS, Warbasse LH: Effects of ciprofloxacin on testosterone and cortisol concentrations in healthy males. Antimicrob Agents Chemother 1989;33:1875-1877.

23. Ho G, Tierney MG, Dales RE: Evaluation of the effect of norfloxacin on the pharmacokinetics of theophylline. Clin Pharmacol Ther 1988;44:35-38. 